\title{
Fast neutron counting in a mobile, trailer-based search platform
}

\author{
Jason P. Hayward ${ }^{1,2,}$, John Sparger ${ }^{1,2}$, Lorenzo Fabris ${ }^{2}$, and Robert J. Newby ${ }^{2}$ \\ ${ }^{1}$ University of Tennessee, Nuclear Engineering Department, 211 Pasqua Engineering Bldg, 37996 Knoxville, USA \\ ${ }^{2}$ Oak Ridge National Laboratory, One Bethel Valley Rd, P.O. Box 2008, MS-6010, 37931 Oak Ridge, USA
}

Received: 19 July 2017 / Received in final form: 20 September 2017 / Accepted: 7 November 2017

\begin{abstract}
Trailer-based search platforms for detection of radiological and nuclear threats are often based upon coded aperture gamma-ray imaging, because this method can be rendered insensitive to local variations in gamma background while still localizing the source well. Since gamma source emissions are rather easily shielded, in this work we consider the addition of fast neutron counting to a mobile platform for detection of sources containing $\mathrm{Pu}$. A proof-of-concept system capable of combined gamma and neutron coded-aperture imaging was built inside of a trailer and used to detect a ${ }^{252} \mathrm{Cf}$ source while driving along a roadway. Neutron detector types employed included EJ-309 in a detector plane and EJ-299-33 in a front mask plane. While the ${ }^{252} \mathrm{Cf}$ gamma emissions were not readily detectable while driving by at $16.9 \mathrm{~m}$ standoff, the neutron emissions can be detected while moving. Mobile detection performance for this system and a scaled-up system design are presented, along with implications for threat sensing.
\end{abstract}

\section{Introduction}

The capability to use radiological and nuclear $(\mathrm{RN})$ search assets to clear a particular area of a city from potential threats is deemed important in the United States. These assets include trailer-based sensors [1] and wearable sensors, and they may also be used for intelligence-based search or chokepoint monitoring. Considering trailer-based systems, while non-imaging systems have been demonstrated with the use of advanced algorithms to be highly sensitive for RN detection during search [2-4], they are not able to discern on which side of a roadway a source is located. On the other hand, a coded-aperture-based system can perform this task while also sensitively and specifically detecting $\mathrm{RN}$ threats $[1,5-8]$. In coded-aperture imaging [9], incident radiation is modulated by a mask before being absorbed in a detector plane, and reconstruction using the modulation pattern allows for imaging of a point source.

In this work, use of fast neutron counting for neutron source detection was demonstrated on board a proof-ofconcept $(\mathrm{PoC})$ platform called the Dual hybrid detectionlocalization-identification (DLI) system at Oak ridge national laboratory (ORNL). The system is sensitive to both gammas and neutrons [10,11], is composed of a hybrid combination of radiation sensing materials, and can perform functions of RN source detection, localization, and identification.

\footnotetext{
* e-mail: jhayward@utk.edu
}

Since gamma source emissions are rather easily shielded, in this work we consider the addition of fast neutron counting and imaging to a mobile platform for detection of sources containing $\mathrm{Pu}$. The fast neutron counting is designed to enable mobile detection of shielded $\mathrm{Pu}$, while the imaging enables localization of said sources. The dual hybrid DLI system, capable of combined gamma and neutron codedaperture imaging, was built inside of a trailer and used to measure ${ }^{137} \mathrm{Cs}$ and ${ }^{252} \mathrm{Cf}$ sources while driving along a roadway. Mobile detection performance for this system and the expectations for a scaled-up system design are discussed.

\section{Experimental setup}

The built PoC (see Fig. 1) is a scaled-down version of a larger, trailer-based system designed for double-sided source search (see Fig. 2). The PoC contains a partially instrumented central detector plane and one of two partially instrumented mask planes.

The central detector plane of the $\mathrm{PoC}$ contains all twenty-one 2 -in-thick $\times 4$-in-wide $\times 16$-in-tall $\mathrm{NaI} \quad(\mathrm{Tl})$ detectors on the bottom row and only five out of twentyone $9.4 \mathrm{~cm}$-thick $\times 9.4 \mathrm{~cm}$-wide $\times 30 \mathrm{~cm}$-tall EJ-309 liquid scintillators on the top row. A fully instrumented detector plane consists of $0.87 \mathrm{~m}^{2}$ of inorganic scintillator and $0.59 \mathrm{~m}^{2}$ of organic scintillator. While the NaI (Tl) detectors were read out by PMTs on a single side, the EJ-309 detectors (Scionix V94 A300/3(2)M) were readout out at each end by Hamamatsu R6233-100 PMTs in an effort to improve pulse shape discrimination performance 

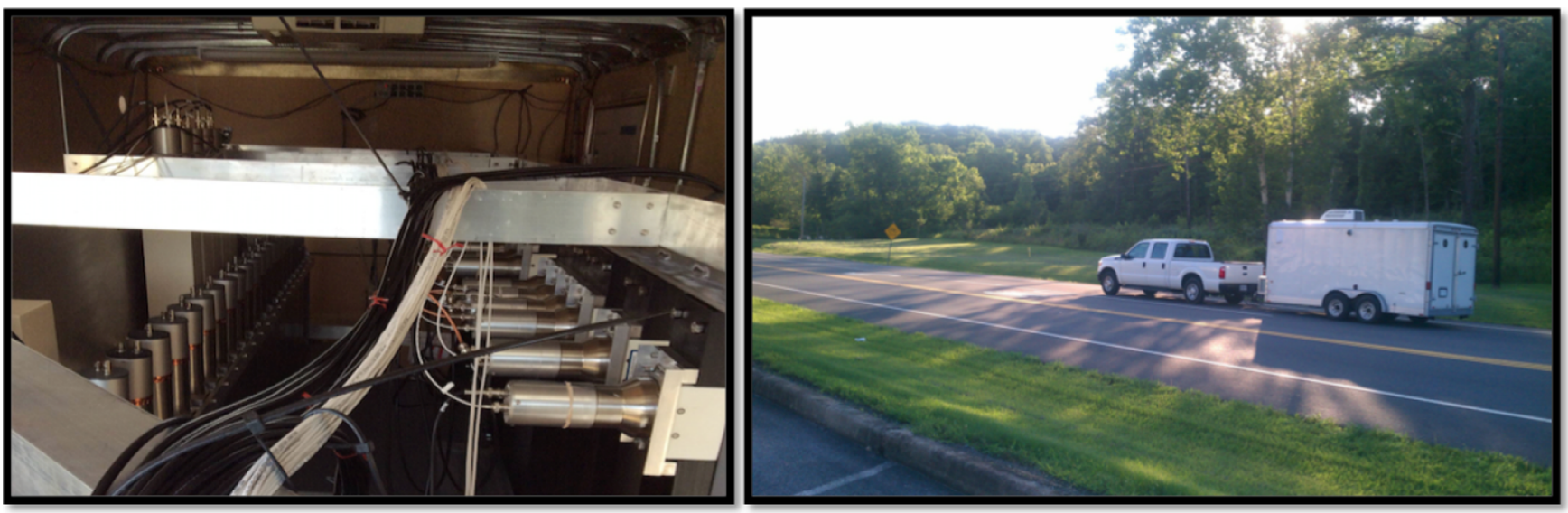

Fig. 1. The photo at left shows our proof-of-concept system (detector plane at left and mask plane at right) built inside a trailer. The photo at right shows the trailer being driven during mobile testing at ORNL.

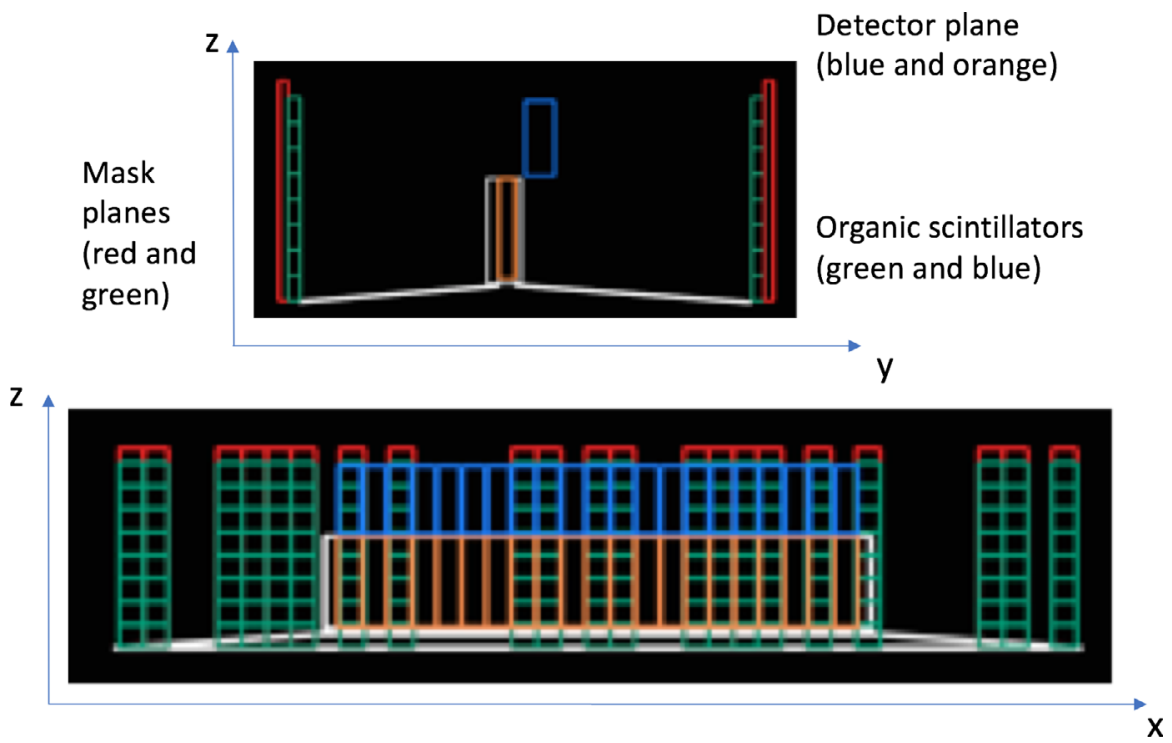

Fig. 2. At top is a side view drawing of a full-scale, trailer-based system designed for double-sided gamma and neutron source search. This is the view that would be seen from the front (or back) of the trailer. A central detector array consists of organic scintillator on top (blue) and inorganic scintillator on bottom (orange). The lateral offset between the different scintillator types allows room for PMT readout for each detector. On either side of the trailer, there exists a dual layered mask made of lead (red) and organic scintillator (green). Additional lead (white) is added to reduce the gamma background. At bottom is the view that one would see standing in between the detector plane and a mask plane, facing the detector plane or toward the side of the trailer.

for such large cells. The centers of the $\mathrm{NaI}(\mathrm{Tl})$ detectors (width dimension) were aligned with the centers of the EJ309 detectors.

The $4.2 \mathrm{~m}$-long mask plane is comprised of a $3 \mathrm{~cm}$-thick layer of high density material to modulate gammas followed by a $4 \mathrm{~cm}$-thick layer of low density material to module fast neutrons. The configuration of the 1D codedaperture mask was two rank 19, uniformly-redundant array patterns [5] tiled side-by-side for increased field-ofview. The mask element widths were $\sim 4$ inches. We used $3 \mathrm{~cm}$-thick lead for the high-density layer and a mixture of $4 \mathrm{~cm}$-thick high-density polyethylene or EJ-299-34 organic scintillator for the second layer. The single row of organic scintillators (a quantity of 20) used were $4 \mathrm{~cm}$-thick $\times 9.75 \mathrm{~cm}$-wide $\times 9.75 \mathrm{~cm}$-tall and were read out by Hamamatsu R6233-100 PMTs (Scionix Model 570). The focal length (mask-detector separation) was $72 \mathrm{~cm}$, creating a fully coded field-of-view of about $110^{\circ}$. This results in $8.5^{\circ}$ angular resolution for gammas or neutrons, or about $10 \mathrm{~m}$ wide pixels at $70 \mathrm{~m}$ standoff. The functionality of the gamma coded aperture capability has been checked as a part of separate work.

The detectors are read out by Struck SIS $3316250 \mathrm{MS} / \mathrm{s}$ 14-bit waveform digitizers. These were run using an embedded Intel Core I7 VME Controller with 2 TB SSD removable storage. The organic scintillators were powered by CAEN 6533 and CAEN AG536SP high voltage power supplies, respectively. A Doppler fifth wheel (Delta DRS1000) 


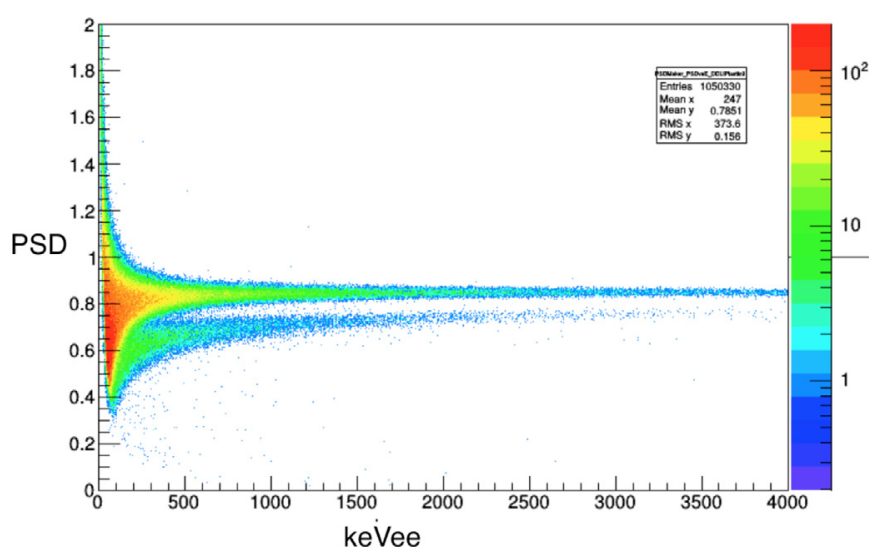

Fig. 3. Example PSD plot from an EJ-299-33 organic scintillator showing the fast fraction of the light as a function of scintillator light output. The gamma band is on top, and the neutron band is below it.

was used to measure vehicle speed, and a Real Time Kinematic GPS (ashtech ProMark 100) was used to ascertain the precise locations of both the trailer and a source.

As described in more detail in [12], gain matching was performed using a ${ }^{137} \mathrm{Cs}$ source in order to equalize the response of the detectors. The pulse shape discrimination in the organic scintillator detectors was studied using a ${ }^{252} \mathrm{Cf}$ source. A standard $Q_{\text {tail }} / Q_{\text {total }}$ method was employed. Neutrons were selected in the organic scintillators by using a $5 \sigma$ cut below the gamma ray band, considering an energy window of $200 \mathrm{keVee}$ to $1000 \mathrm{keVee}$. Additional detail about the experiments conducted is given in [12].

\section{Results}

Figure 3 shows an example PSD plot for one of our organic scintillators. At $200 \mathrm{keVee}$ (or $100 \mathrm{keVee}$ ), the figure-of-merit for our twenty, $4 \mathrm{~cm}$-long EJ-299-34 detectors was found to be $1.78 \pm 0.30$ (or $0.99 \pm 0.14$ ) while it was determined to be $1.34 \pm 0.17$ (or $0.66 \pm 0.07$ ) for our double-ended, $30 \mathrm{~cm}$-long EJ-309 detectors. The poorer performance for the EJ-309 detectors is due to the detector length.

Measurements where the $\mathrm{PoC}$ system was moving past a ${ }^{252} \mathrm{Cf}$ source were taken on a section of Bethel Valley Rd at ORNL. Background data was also taken with no source present. The strength of the ${ }^{252} \mathrm{Cf}$ source was measured at ORNL with a shuffler to be $279000 \mathrm{n} / \mathrm{s}$. At closest approach, the PoC trailer passed by $15.9 \mathrm{~m}$ away from the source, corresponding to an optimal counting window length [3] of $43.8 \mathrm{~m}$. The neutron counts were mapped onto the $2 \mathrm{D}$ location of the measurement to obtain Figure 4, showing that the neutron source is clearly visible. The same type of plot (not shown) of the ${ }^{252} \mathrm{Cf}$ gamma counts showed no source visible because the source strength is small compared with the gamma background and its local variations.

The neutron background, on the other hand, in this area can be assumed to be constant over a few hours of measurement on any particular day. Under this assumption, all the background data collected with no source present was used to calculate the mean neutron background. Next, the neutron source counts in a $43.8 \mathrm{~m}$ long

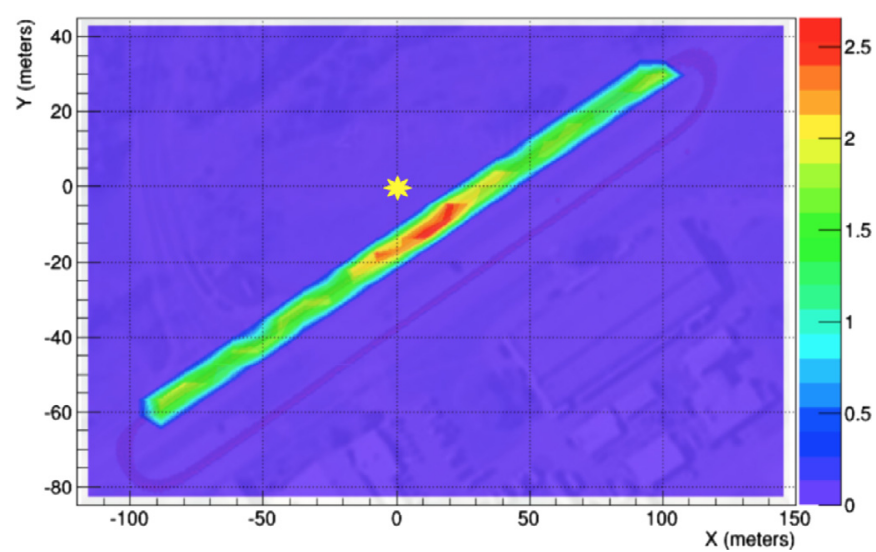

Fig. 4. Measured fast neutron count rate data from passing a ${ }^{252} \mathrm{Cf}$ source (yellow star) is superimposed on top of map.

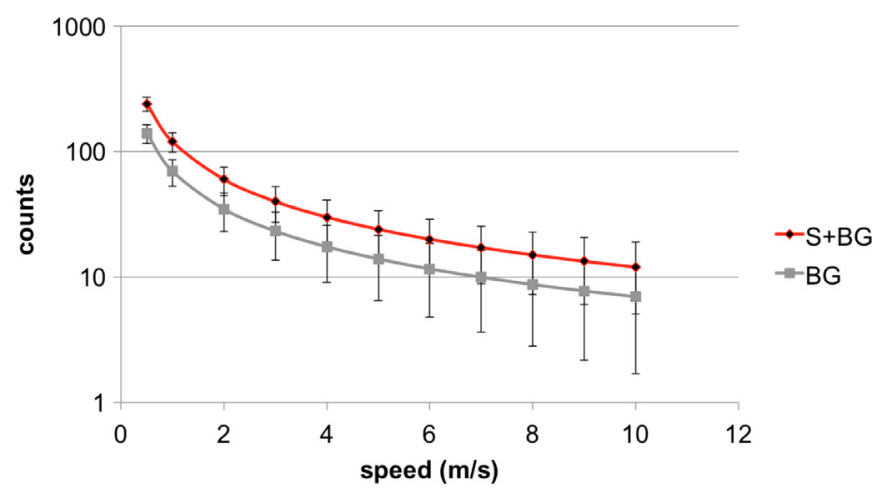

Fig. 5. Fast neutron counting data for the source $(\mathrm{S})$ and background (BG) from all 25 detectors $(200-1000 \mathrm{keVee})$ in PoC trailer as it passes the ${ }^{252} \mathrm{Cf}$ source at various speeds. Error bars indicate $\pm 2 \sigma$.

window about the source were tallied. The results are shown in Figure 5. With so few detectors, the source is not very well separated from the background given this neutron cut (200-1000 keVee).

Next, we considered the effect of scaling up the $\mathrm{PoC}$ design, having only $6 \%$ of the EJ-299-33 scintillators (in one mask) and $24 \%$ of the EJ-309 scintillators compared to the designed full system (refer to Fig. 2). This change increases the width of the system from $0.76 \mathrm{~m}$ to $1.51 \mathrm{~m}$. Considering the effects on both background and source counts, detection results for the scaled-up system are shown in Figure 6. At $4 \sigma$ confidence, the scaled-up system might be expected to be capable of detecting an $O\left(10^{5} \mathrm{n} / \mathrm{s}\right)$ source almost $100 \%$ of the time at speeds up to $5 \mathrm{~m} / \mathrm{s}(\sim 11 \mathrm{mph})$.

\section{Conclusions}

The built proof-of-concept system, designed to detect, locate and identify a broad set of radiological and nuclear threat sources, demonstrates that a compact design for achieving dual gamma and fast neutron coded-aperture imaging is possible. In this work, we show that such a system is sensitive to a $2.75 \times 10^{5}$ fast neutron source while driving by it at speed, even with non-optimal PSD 

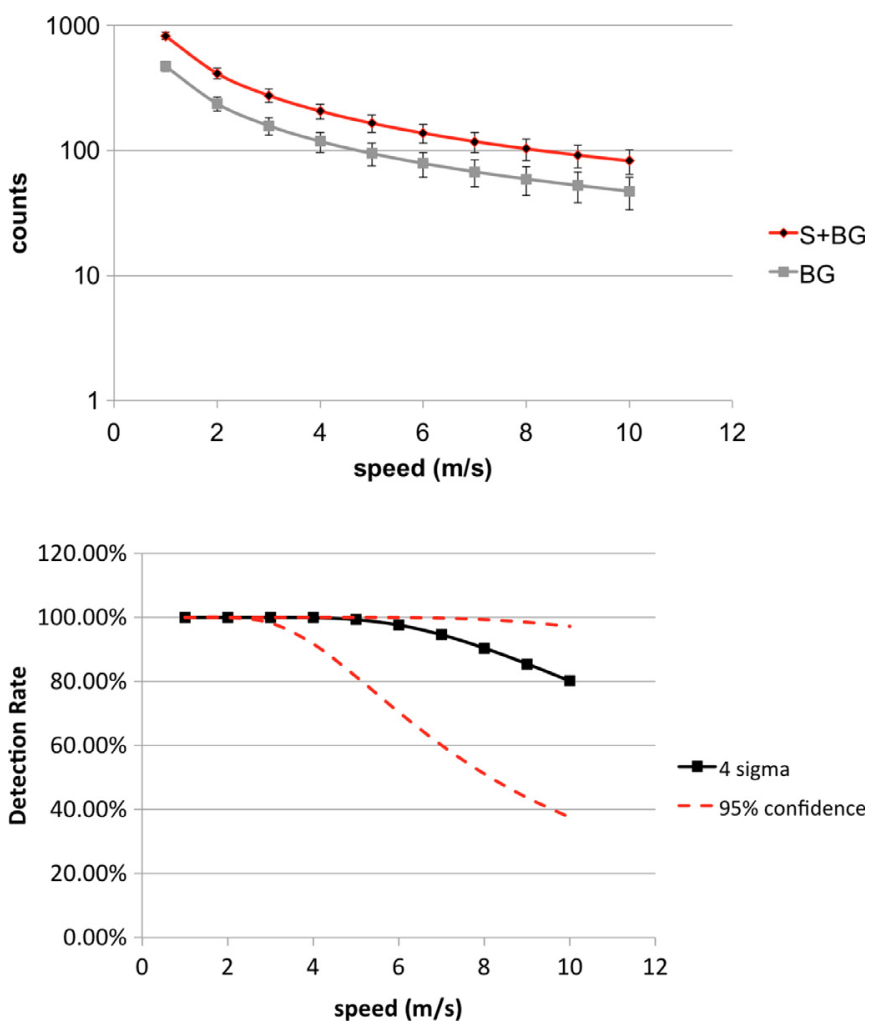

Fig. 6. Expected fast neutron counting data (top) and expected source detection rate (bottom) for the full-scale system (see Fig. 2) as it passes the ${ }^{252} \mathrm{Cf}$ source at various speeds. Error bars indicate $\pm 2 \sigma$.

performance. Further improvements in PSD, dropping the lower cut from our reported value of $200 \mathrm{keVee}$, are expected to improve the fast neutron detection efficiency at a given level of confidence. We suggest that the measurement results presented here are representative of the fact that some sources (in the subset of shielded $\mathrm{Pu}$ threats) could be visible while moving only through use of the fast neutron counting modality. This work implies that the addition of fast neutron counting, especially considering a brighter organic scintillator like stilbene [13], may add value for mobile detection of shielded $\mathrm{Pu}$. The full-scale mobile system proposed here, although expected to add capability, is quite complex, expensive, and heavy.

This work has been sponsored by the Domestic Nuclear Detection Office of the Department of Homeland Security under IAAs HSHQDC10X00662 and HSHQDC13X000172. This support does not constitute an express or implied endorsement on the part of the Government. The authors would also like to thank Klaus Ziock for helpful discussions contributing toward the design of this system.

\section{References}

1. K.P. Ziock, L. Fabris, D. Carr, J. Collins, M. Cunningham, F. Habte, T. Karnowski, W. Marchant, A fieldable-prototype, large-area, gamma-ray imager for orphan source search, IEEE TNS 55, 3643 (2008)

2. L.J. Mitchell, B.F. Phlips, W.N. Johnson, E.A. Wulf, A.L. Hutcheson, C.J. Lister, K.D. Bynum, B.E. Leas, G. Guadagno, Mobile imaging and spectroscopic threat identification (MISTI): system overview, in Nuclear Science Symposium Conference Record (NSS/MIC) (IEEE, 2009), p. 110

3. T.J. Aucott, D.H. Chivers, K. Vetter, Proximity localization with the mobile imaging and spectroscopic threat identification (MISTI) system, in IEEE Nuclear Science Symposium Conference Record (2011)

4. T.J. Aucott, M.S. Bandstra, V. Negut, J.C. Curtis, D.H. Chivers, K. Vetter, In effects of background on gamma-ray detection for mobile spectroscopy and imaging systems, IEEE TNS 61, 985 (2014)

5. K.P. Ziock, J.W. Collins, L. Fabris, S. Gallagher, B.K.P. Horn, R.C. Lanza, N.W. Madden, Source-search sensitivity of a large-area, coded-aperture, gamma- ray imager, IEEE TNS 53, 1614 (2006)

6. F. Habte, M.F. Cunningham, L. Fabris, K.P. Ziock, Performance of a large-area, gamma imager using a point source injection technique, in Nuclear Science Symposium Conference Record (2007), p. 1157

7. R.D. Penny, W.E. Hood, R.M. Polichar, F.H. Cardone, L.G. Chavez, S.G. Grubbs, B.P. Huntley, R.A. Kuharski, R.T. Shyffer, L. Fabris, A dual-sided coded-aperture radiation detection system, Nucl. Instrum. Methods Phys. Res. A 652, 578 (2011)

8. A. Hoover, M. Wallace, M. Galassi, M. Mocko, D. Palmer, L. Schultz, S. Tornga, Simulation and modeling for the stand-off radiation detection system (sords) using geant4, in Nuclear Science Symposium Conference Record (NSS/MIC), IEEE (2009), p. 914

9. E.E. Fenimore, T.M. Cannon, Coded aperture imaging with uniformly redundant arrays, Appl. Opt. 17, 337 (1978)

10. B. Ayaz-Maierhafer, J.P. Hayward, K.P. Ziock, M.A. Blackston, L. Fabris, Transmission and signal loss in mask designs for a dual neutron and gamma imager applied to mobile standoff detection, Nucl. Instrum. Methods Phys. Res. A 712, 1 (2013)

11. B. Ayaz-Maierhafer, J.P. Hayward, K.P. Ziock, M.A. Blackston, L. Fabris, Angular resolution study of a combined gamma-neutron coded aperture imager for standoff detection, Nucl. Instrum. Methods Phys. Res. A 712, 120 (2013)

12. J.E. Sparger, Machine Learning and Neutron Sensing in Mobile Nuclear Threat Detection, Ph.D. diss., University of Tennessee, 2016. http://trace.tennessee.edu/utk_graddiss/ 3749

13. N. Zaitseva, A. Glenn, L. Carman, H.P. Martinez, R. Hatarik, H. Klapper, S. Payne, Scintillation properties of solution-grown trans-stilbene single crystals, Nucl. Instrum. Methods Phys. Res. A 789, 8 (2015)

Cite this article as: Jason P. Hayward, John Sparger, Lorenzo Fabris, Robert J. Newby, Fast neutron counting in a mobile, trailer-based search platform, EPJ Nuclear Sci. Technol. 3, 35 (2017) 